\title{
EDITORIAL
}

\section{Editorial for 2015}

NPG Asia Materials (2015) 7, e160; doi:10.1038/am.2015.1; published online 20 February 2015

\section{This article has been corrected since Online Publication and an erratum is also published.}

Last year has seen further growth of the journal both in terms of quality and quantity of the submissions, with the impact factor reaching 9.902. In addition, Research Highlights have been added to the journal's content to report on the latest trends in materials science published in other journals. All this development would not be possible without the invaluable contributions from our authors, reviewers, Advisory Board members and Editors to whom we all want to express our thanks.

A large part of the growth of the journal was carried by scientific contributions from materials researchers in China. In 2014 alone, articles written by authors of Chinese institutions comprised about $40 \%$ of the journal's original contents, and Chinese scientists also figure prominently among the journal readership. For this year's Editorial I have asked a leading Chinese scientist, Lei Jiang from the Institute of Chemistry in Beijing, to share with us his view and outlook of the field in his country (MV).

\section{CURRENT STATE AND TRENDS IN MATERIALS RESEARCH IN CHINA}

China has set up a base after a thriving decade of materials research for more original and innovative basic science and applied technology

With rapid development in materials science in the past decade, some remarkable achievements have been on display to the world. China has placed herself as one of the frontier countries of materials science and technology owing to her fast progress in the field. From unique rare earth materials, carbon-based materials, nano-biomaterials, conjugated polymeric materials to superconductive materials, Chinese scientists have made some of the most notable achievements in basic science and applications in the worldwide competition for development of advanced materials. I would draw attention to two notable trends in the development of materials science in China. One is a shift in research evaluation from quantity of publications to quality that becomes more and more important. The other is that the portion of true innovation and original technology has remarkably increased in the past decade. These changes are closely related to the large investments and programs from the Chinese government, and the innovative and hard work of Chinese scientists as well.

Especially, in the past decade excellent overseas returnees have brought rapid development and dynamics into the Chinese research community. Importantly, these returnees went beyond the advanced knowledge of modern science and technology they imbibed from the Western countries by further combining ancient Chinese philosophy with the Western research mode of thinking. Thus, they have stimulated remarkable development of science in China in its own way. Of the ancient Chinese philosophy, the yin and yang is the essence and core, reflecting the unity of opposite. ${ }^{1}$ It has been constantly proved in a wide field of research areas, from materials science to politics and economy (see Figure 1). In addition to the yin and yang philosophy, 'the holographic mode of thinking,', which can provide the whole information from a miniature part of an object, created the ancient resplendent culture and science of China. However, in the past 200 years, emphasis on the experience and heritage gradually caused science and technology in China to lag behind other countries and to drop to the lowest level. Fortunately, many excellent young scientists have come back to China and helped the country to gradually catch up with the research developments in the rest of the world. As a result, the Chinese scientists have been gradually shifting away from follow-up studies and building up their own logo in the research field. They have been following an ancient Chinese principle that has been revived by the country's modern leaders, to 'Seek truth from facts' (实事求是). ${ }^{3}$ 'Facts' represent the existing reality and problems, 'seek' is to explore and solve, and 'truth' is the knowledge of the basic phenomenon. Later, Chairman Mao Zedong himself also proposed how to achieve the principle using three terms, 'Discovery', 'Invention' and 'Creation'. Let me take our recent study on bio-inspired surface with superhydrophobicity as an example. ${ }^{4}$ We observed the superhydrophobicity of lotus leaves and revealed its mechanism that originates from the unique surface roughness and a wax layer. This is the first step of 'Discovery'. Subsequently, using the knowledge of the basic mechanism we could fabricate artificial superhydrophobic surfaces from different materials. This represents the second step of 'Invention'. Finally, we went beyond the above and made a creative smart switch that can reversibly convert between superhydrophobicity and superhydrophilicity. This is the third step of 'Creation'. Of course, the principle of 'Seeking truth from facts' has also been widely applied in other fields and undoubtedly led to great successes, examples of which are mesoporous materials, nanoalloys, carbon nanotubes embedded with nanocatalyst, superplastic copper films and so on. This process of 'Discovery', 'Invention' and 'Creation' is not a closed cycle, but is constantly evolving in a helical way. The principle has constantly run through the whole developing history of science, technology and basic knowledge. 


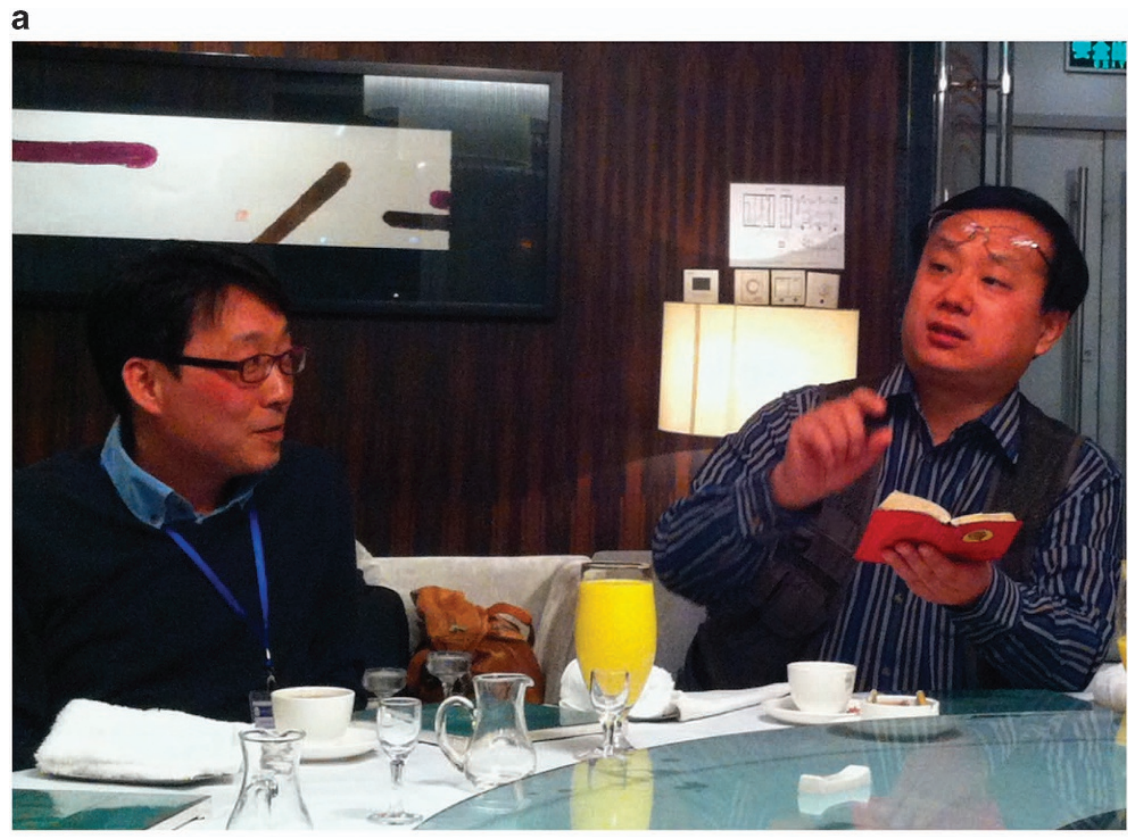

b

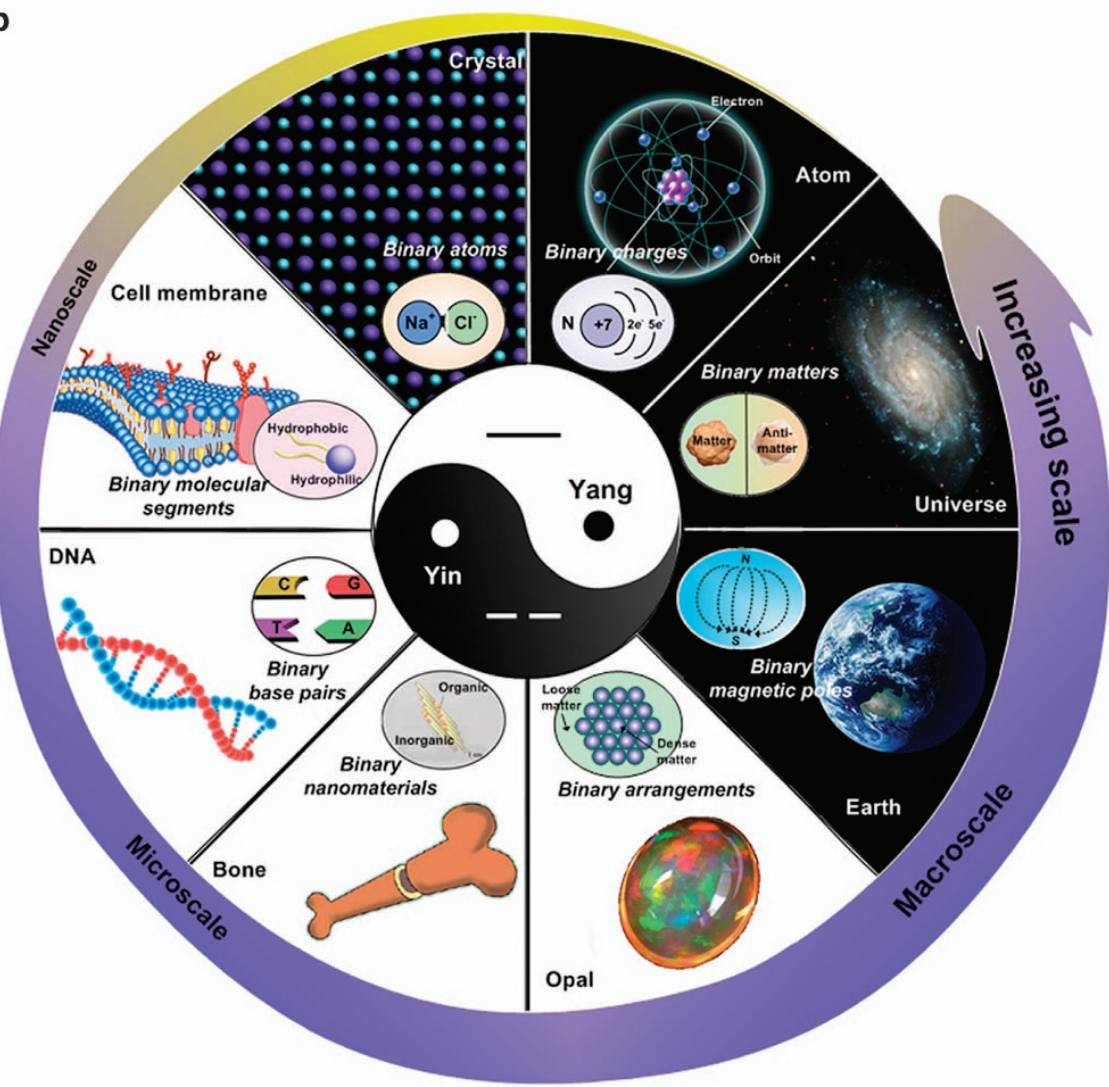

Figure 1 (a) Professor Lei Jiang (at right) is explaining the principle of 'Seeking truth from facts' (实事求是) to the Associate Editor Hee Cheul Choi (at left) It includes three steps: 'Discovery', 'Invention' and 'Creation'. (b) The combination of the Chinese yin-yang philosophy and western research thinking has been widely applied into the various research fields in science and technology, not limited to materials science. ${ }^{5}$

To my great pleasure, I see more and more Chinese researchers contributing to the solution of global scientific and technical challenges, together with the Asia-Pacific and other regions. We believe that the basic principle of 'Seeking truth from facts' will help China have a bright future in materials science and technology. The research philosophy is not limited only to China but also has been widely affecting the Asian countries. We believe that the global center of science and technology would gradually migrate to the Asian region in 20 or 30 years (LJ). 
Martin Vacha ${ }^{1}$ and Lei Jiang ${ }^{2}$

${ }^{1}$ Department of Organic and Polymeric Materials, Tokyo Institute of Technology, Tokyo, Japan and ${ }^{2}$ Institute of Chemistry, Chinese Academy of Sciences, Beijing, China E-mail: vacha.m.aa@m.titech.ac.jp

1 Legge, J. The I Ching: The Book of Changes (Dover Publication, Inc., New York, NY, USA, 1963).

2 Gabor, D. A new microscopic principle. Nature 161, 777-778 (1948).

3 Zhuang, Z. Book of Han. AD 111.

4 Tian, Y., Su, B. \& Jiang, L. Interfacial material system exhibiting superwettability. Adv. Mater. 26, 6872-6897 (2014).
5 Su, B., Guo, W. \& Jiang, L. Learning from nature: binary cooperative complementary nanomaterials. Small (e-pub ahead of print 29 July 2014; doi:10.1002/ smll.201401307).

(c) (1) This work is licensed under a Creative Commons Attribution 4.0 International License. The images or other third party material in this article are included in the article's Creative Commons license, unless indicated otherwise in the credit line; if the material is not included under the Creative Commons license, users will need to obtain permission from the license holder to reproduce the material. To view a copy of this license, visit http:// creativecommons.org/licenses/by/4.0/ 


\section{ERRATUM}

\section{Editorial for 2015}

\section{Martin Vacha and Lei Jiang}

NPG Asia Materials (2015) 7, e177; doi:10.1038/am.2015.31; published online 24 April 2015

Correction to: NPG Asia Materials (2015) 7, e160; doi:10.1038/ am.2015.1; published online 20 February 2015

After publication of this article, it has come to our attention that there is a formatting error in this article. Header text in the article was not properly inserted. With publication of this erratum we have made correction to the article in both PDF and HTML versions. The error has now been rectified.

The publisher would like to apologize for any inconvenience caused. 